\title{
Analysis of the Ferromagnetic Transition in Melt- Spun Gadolinium Nanocrystals
}

\author{
J.G. Bohnet and P. M. Shand \\ Department of Physics \\ University of Northern lowa \\ Cedar Falls, lowa 50614-0150 USA \\ J. Goertzen and J.E. Shield \\ Department of Mechanical Engineering \\ and Nebraska Center for Materials and Nanoscience \\ University of Nebraska-Lincoln \\ Lincoln, Nebraska 68588 USA \\ D. Schmitter, G. Shelburne and D. L. Leslie-Pelecky \\ Department of Physics \& Astronomy \\ and Nebraska Center for Materials and Nanoscience \\ University of Nebraska-Lincoln \\ Lincoln, Nebraska 68588 USA
}

Received:June 29, $2007 \quad$ Accepted: August 19, 2007

\begin{abstract}
Magnetic materials are present in rewriteable disk drives, electric motors and generators, and signal transformers/receivers. To improve the performance of these and other devices, much research in magnetism continues to be done. In particular, materials that are disordered on the atomic and nanometer scales have recently been the subject of extensive research, as the arrangement of atoms and the interactions between them significantly affect a material's magnetic properties. We have prepared a disordered pure gadolinium (Gd) system using a meltspinning technique. This resulted in a system of $\mathrm{Gd}$ crystals on the order of $160 \mathrm{~nm}$ in size embedded in an amorphous Gd matrix. The structure was identified using X-ray analysis and transmission electron microscopy. AC susceptibility and DC magnetization measurements at various temperatures $(280-350 \mathrm{~K})$ and $\mathrm{DC}$ bias fields $(0-3 \mathrm{kOe})$ were performed on a sample of the nanocrystalline Gd. Using modified Arrott-Noakes plots and scaling ideas for a second-order phase transition, critical exponents and the Curie temperature $\left(T_{C}\right)$ for the ferromagnetic transition in the nanocrystalline Gd system were obtained. $T_{C}$ was found to be $289.70 \mathrm{~K}$, and the critical exponents had shift away from those of bulk Gd and toward those of the Heisenberg model with short-range interactions, indicating that melt-spinning suppressed the interactions present in bulk Gd.
\end{abstract}

\section{INTRODUCTION}

The arrangement of atoms in a material and the interactions between them significantly affect a material's magnetic properties. Introducing disorder into a system affects this arrangement, and thereby affects the material's properties. An extreme example of disorder is an amorphous material, in which there is no crystal lattice structure. The magnetic properties of many amorphous ferromagnets have been studied, including alloys containing gadolinium [1-6]. In this paper, we present experimental data on the magnetic properties of nanostructured gadolinium (Gd). The nanostructures we have studied consist of nanometer-sized crystallites or 
grains embedded in an amorphous $\mathrm{Gd}$ background, as indicated by $\mathrm{x}$-ray analysis and transmission electron microscopy (TEM). The focus of our investigations was the ferromagnetic to paramagnetic transition of nanostructured Gd. AC susceptibility measurements with respect to temperature and DC magnetization measurements with respect to temperature and applied external magnetic field were performed in order to determine the transition temperature and critical exponents, which characterize the nature of the transition.

\section{EXPERIMENTAL}

The samples were prepared using a melt-spinning apparatus at the University of Nebraska-Lincoln. The gadolinium was prepared for melt-spinning by arc-melting under an argon gas atmosphere. The meltspinning was performed under a vacuum to prevent oxidation. The samples obtained were ribbons $7-10 \mathrm{~mm}$ long. The mass of the sample that was analyzed was 11.92 mg. X-ray diffraction analysis indicated that the sample consisted of a nanocrystalline phase $(\approx 75 \%)$ and an amorphous phase $(\approx$ $25 \%$ ). TEM imaging shows the grain size to be approximately $160 \mathrm{~nm}$.

The magnetic measurements were performed on a Quantum Design Physical Property Measurement (PPMS) system, with an AC susceptibility (ACMS) attachment. All AC susceptibility measurements were performed in an AC-driving field with amplitude of $3 \mathrm{Oe}$ and a frequency of $1 \mathrm{kHz}$. In the vicinity of the paramagnetic to ferromagnetic transition, we performed measurements of the AC susceptibility versus temperature $(286 \mathrm{~K}-304 \mathrm{~K})$ with superimposed $D C$ bias fields ranging from 600-3000 Oe in increments of $200 \mathrm{Oe}$. In addition, DC magnetization versus external field data were taken along isotherms at intervals of $1 \mathrm{~K}$ from $286 \mathrm{~K}-294 \mathrm{~K}$ in fields up to 6 Tesla. The data were corrected for demagnetization; the demagnetization factor ( $\mathrm{N}=0.59$ ) was determined using low field measurements of the magnetization versus external field and the density of the sample.

\section{RESULTS}

Ferromagnetic materials that are cooled below a certain critical temperature,
$\mathrm{T}_{\mathrm{C}}$, exhibit long range ordering of their magnetic moments, as the strength of the thermal fluctuations becomes less than that of the magnetic moments' interactions. A phase transition occurs at $T_{C}$, the material going from a paramagnetic state above $T_{C}$ to a ferromagnetic state below $\mathrm{T}_{\mathrm{C}}$. The phase transition region is a useful region to study as the material can be characterized according to its transition temperature and its critical exponents, which are related to parameters such as spontaneous magnetization and initial susceptibility near the transition temperature.

Three important critical exponents for this study are $\beta, \gamma$, and $\delta$. Beta $(\beta)$ is the spontaneous (zero magnetic field) magnetization exponent, which is defined by the following relation:

$$
M_{S}(T)=M_{0}(-\varepsilon)^{\beta}, \varepsilon<0,
$$

where $\varepsilon=\frac{T-T_{c}}{T_{c}}$ and $M_{0}$ is a critical amplitude. Gamma ( $\mathrm{Y}$ ) is the isothermal magnetic susceptibility exponent defined as

$$
\chi_{0}^{-1}(T)=\left(h_{0} / M_{0}\right) \varepsilon^{\gamma}, \varepsilon>0
$$

where $\chi_{0}^{-1}$ is the inverse zero-field susceptibility, and $h_{0}$ is a critical amplitude. Delta $(\delta)$ is the critical isotherm exponent:

$$
M=D H^{\frac{1}{\delta}}, \varepsilon=0
$$

where $H$ is the demagnetization adjusted applied magnetic field, and $D$ is a critical amplitude [7]. Note that Eqn. (1) and (2) are strictly valid in the limit $\varepsilon \rightarrow 0$, i.e., in the asymptotic critical region. Eqn. (3) is valid exactly at $T=T_{C}$. Clearly, the accurate determination of $T_{C}$ is of paramount importance.

\section{a. DC Magnetization}

In this subsection, we present data on the temperature variation of DC magnetization in an applied magnetic field, which were used to determine $T_{C}$ and the critical exponents $\gamma, \beta$, and $\delta$ by ArrottNoakes analysis. The unmodified isotherms for magnetization versus applied field are in Figure 1. 


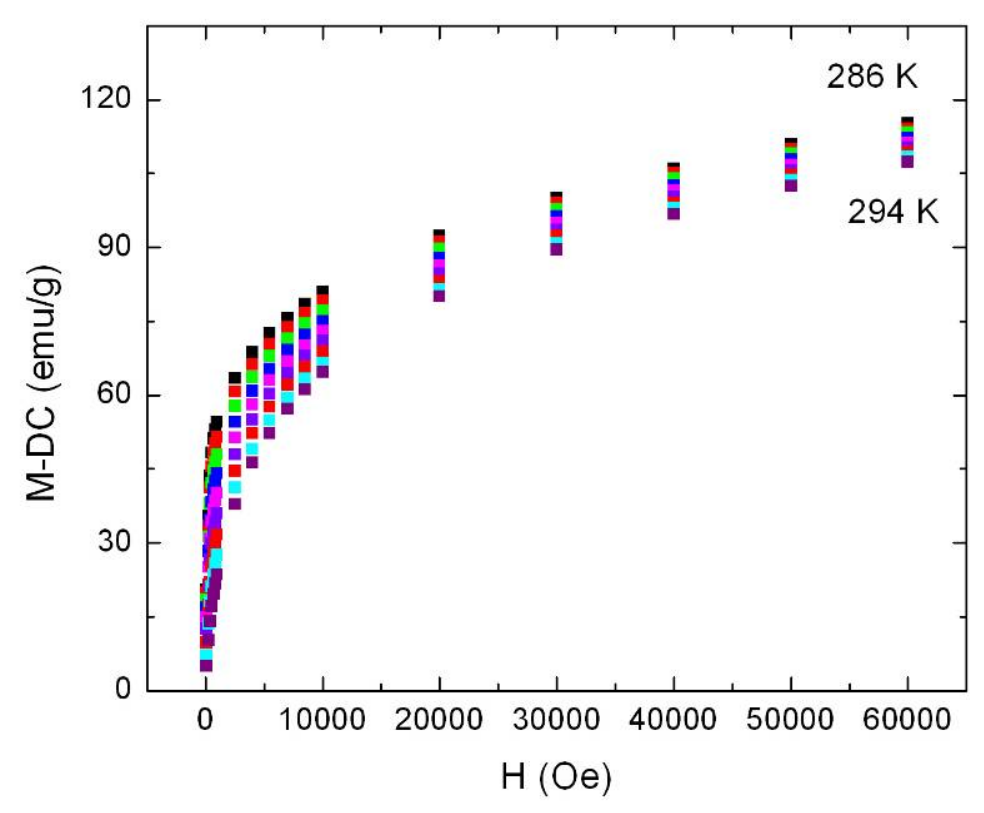

Figure 1. Magnetization versus external field for temperatures 286-294 K.

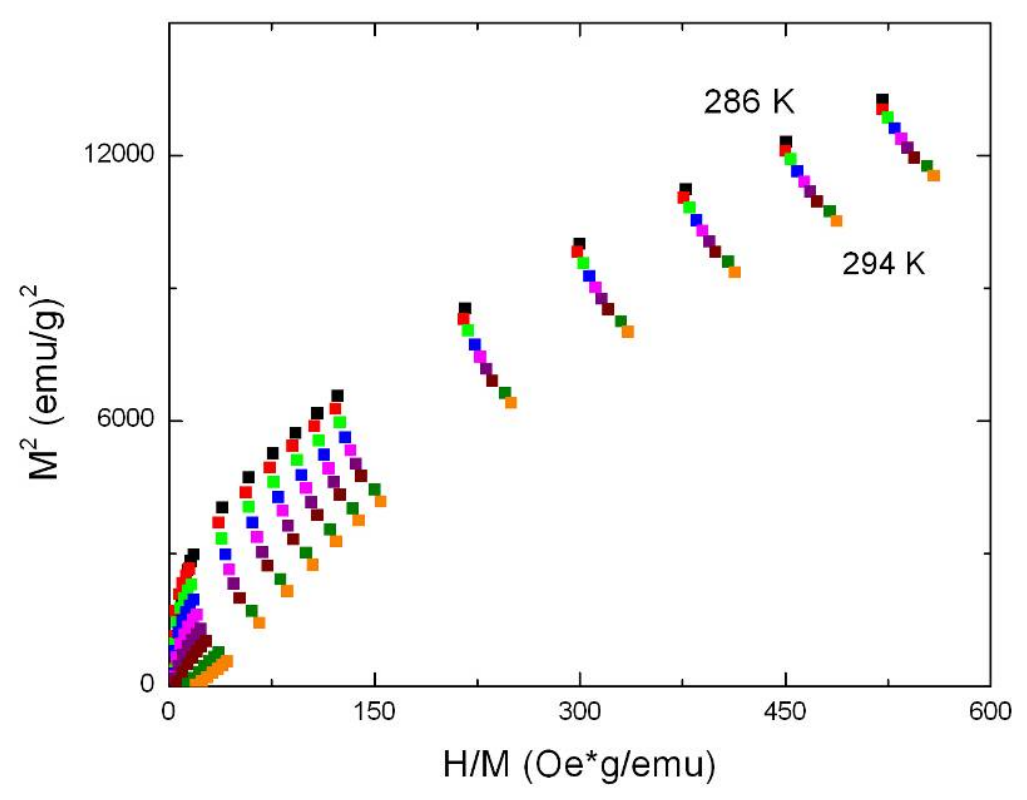

Figure 2. Standard Arrott Plot for temperatures 286 - $294 \mathrm{~K}$.

An Arrott-Noakes plot is a graph of $M^{\frac{1}{\beta}}$ versus $\left(\frac{H}{M}\right)^{\frac{1}{\gamma}}$ that depends on the relationship

$$
\left(\frac{H}{M}\right)^{\frac{1}{\gamma}}=\frac{T-T_{C}}{T_{1}}+\left(\frac{M}{M_{1}}\right)^{\frac{1}{\beta}}
$$

which represents a magnetic equation of state and is consistent with Eqn. (1) and (2) [8]. A standard Arrott plot uses mean- field theory exponents $(\gamma=1, \beta=0.5, \delta=3)$, 
which are characteristic of systems with infinite-range interactions. Thus, the relationship simplifies to a graph of $\mathrm{M}^{2}$ vs. $\mathrm{H} / \mathrm{M}$. When correct exponent values are used in the Arrott-Noakes plot, the data has a linear relationship for sufficiently large fields, and the extrapolations to the axes yields the values of spontaneous magnetization $M_{S}$ and inverse zero-field susceptibility $X_{0}^{-1}$. Also note that for the isotherm at $\mathrm{T}=\mathrm{T}_{\mathrm{C}}$, the $\mathrm{y}$-intercept should be zero.

The standard Arrott plot in Figure 2 is clearly non-linear at all fields, indicating that mean field theory does not apply, and the sample has shorter range interactions influencing the transition.

In order to determine correct values for $\gamma$ and $\beta$, Eqn. (4) was rearranged so that a nonlinear curve fitting routine could be performed on the data with $\gamma$ and $\beta$ as two of the parameters. To perform the nonlinear fit, the initial values of the critical exponents that were used were those for the classical 3D Heisenberg model with short-range interactions $(\beta=0.365, \gamma=1.386, \delta=4.78)$ [9]. This process yielded preliminary values of $\gamma$ and $\beta$, which were used to generate an Arrott-Noakes plot (Figure 3).

The intercepts were extrapolated and used to determine $T_{C}, M_{S}(T)$, and $X_{0}{ }^{-}$ ${ }^{1}(T)$, which were then used to generate a log-log plot (Figure 4). The slopes of these plots gave updated values of $\gamma$ and $\beta$ [see Eqn. (1) and Eqn. (2)]. The resulting values of $\gamma$ and $\beta$ were then used to reconstruct the Arrott-Noakes plot. This process was followed iteratively until $\gamma$ and $\beta$ were constant within uncertainty limits. The process resulted in a $\beta=0.389 \pm 0.017, \gamma=$ $1.300 \pm 0.014$, and $T_{C}=289.70 \pm 0.16 \mathrm{~K}$.

With an accurate value for $T_{C}$, the critical exponent $\delta$ can be calculated directly from the DC magnetization data on the critical isotherm with a log-log plot of magnetization versus applied field [see (3)]. Since our data was taken at 1 degree intervals, the value of delta was interpolated from the inverse of the slope of the $290 \mathrm{~K}$ and $289 \mathrm{~K}$ isotherms (Figure 5).

From the interpolation, $\delta=4.32 \pm$ 0.02 . Now, critical exponents must obey the Widom scaling relation [10]

$$
\delta=1+\gamma / \beta \text {. }
$$

Inserting the experimental values for $\beta$ and $\gamma$ yields $\delta=4.34 \pm 0.03$. Thus, the Widom scaling relation is satisfied.

b. AC susceptibility

Ac susceptibility data provides an independent means of analyzing the transition and determining the critical exponents. The data in Figure 6 shows how the AC susceptibility varies over the entire temperature range of the instrument. One can see from the inset that the susceptibility falls dramatically near $300 \mathrm{~K}$. This is one indication that a ferromagnetic-paramagnetic transition is taking place.

When a system undergoes a paramagnetic to ferromagnetic transition, the AC susceptibility as a function of temperature with a DC magnetic field superimposed has a peak near the transition temperature. This peak shifts with a change in the applied field, which is a signature of critical fluctuations that occur during a phase transition. The data in Figure 7 confirms that a phase transition is indeed taking place in the nanostructured $\mathrm{Gd}$ sample. The maximum susceptibility was located for each data set, and the temperature at which that maximum occurs was recorded as $\mathrm{T}_{\mathrm{m}}$. At each $T_{m}, \varepsilon_{m}=\left(T_{m}-T_{C}\right) / T_{C}$ was calculated. Now, according to the scaling theory of phase transitions [11], $\varepsilon_{m} \propto H_{i}^{1 /(\gamma+\beta)}$, from which we obtain:

$$
T_{m}=\left(k * T_{C}\right)\left(H^{\frac{1}{\gamma+\beta}}\right)+T_{C} .
$$

The data in Figure 8 show that $T_{C}$ using this relation is $290.2 \pm 0.7 \mathrm{~K}$, agreeing within uncertainty limits with the $T_{C}$ from the $D C$ magnetization data. The values for $\gamma$ and $\beta$ were taken from the best values obtained from the Arrott-Noakes analysis.

The AC susceptibility data can also be used to determine the exponent $\gamma$. Scaling arguments yield the relation

$$
\chi^{\prime}\left(h, T_{m}\right) \propto \varepsilon_{m}^{-\gamma} .
$$




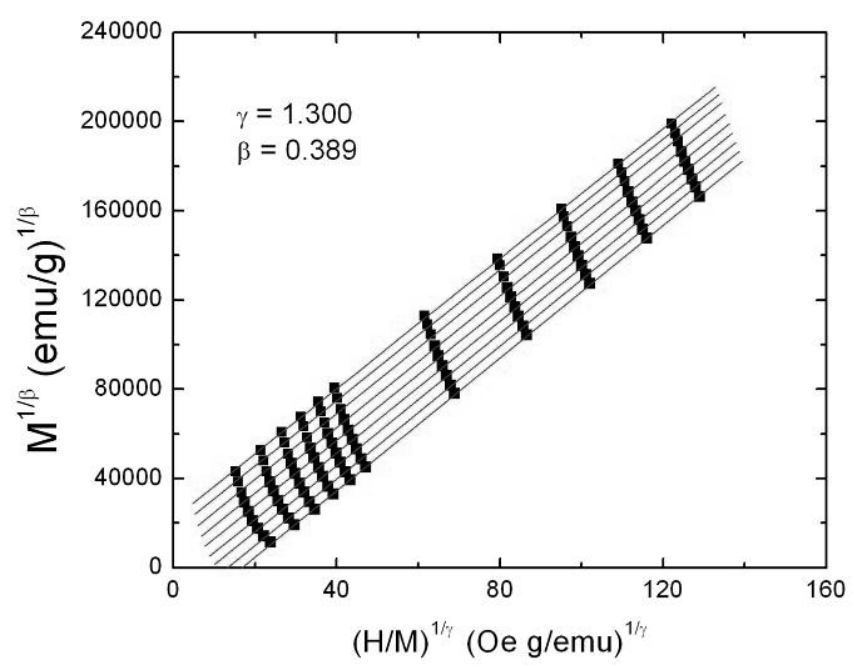

Figure 3. Arrott-Noakes plot with the same data used to generate the Arrott plot in Figure 2.

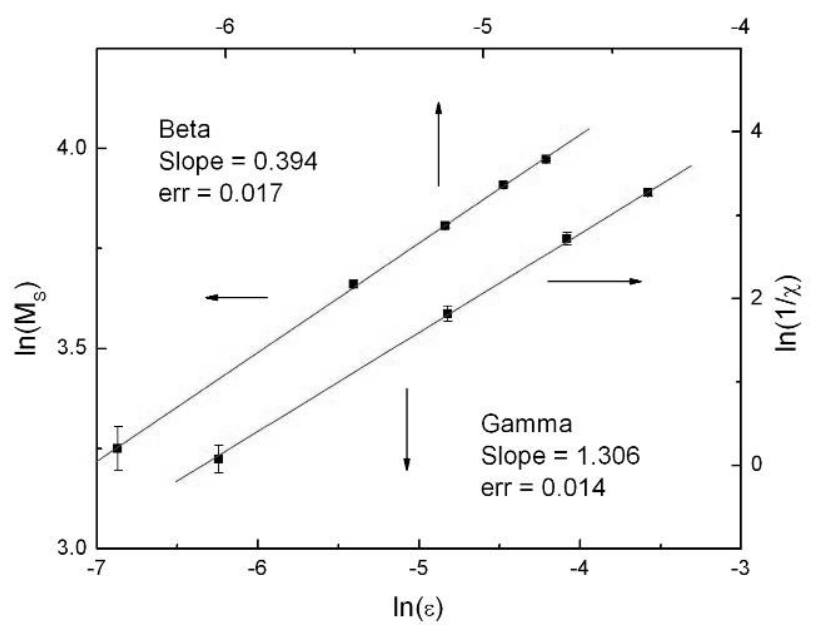

Figure 4. Double logarithmic plots for the spontaneous magnetization and inverse susceptibility to determine critical exponents $\gamma$ and $\beta$.
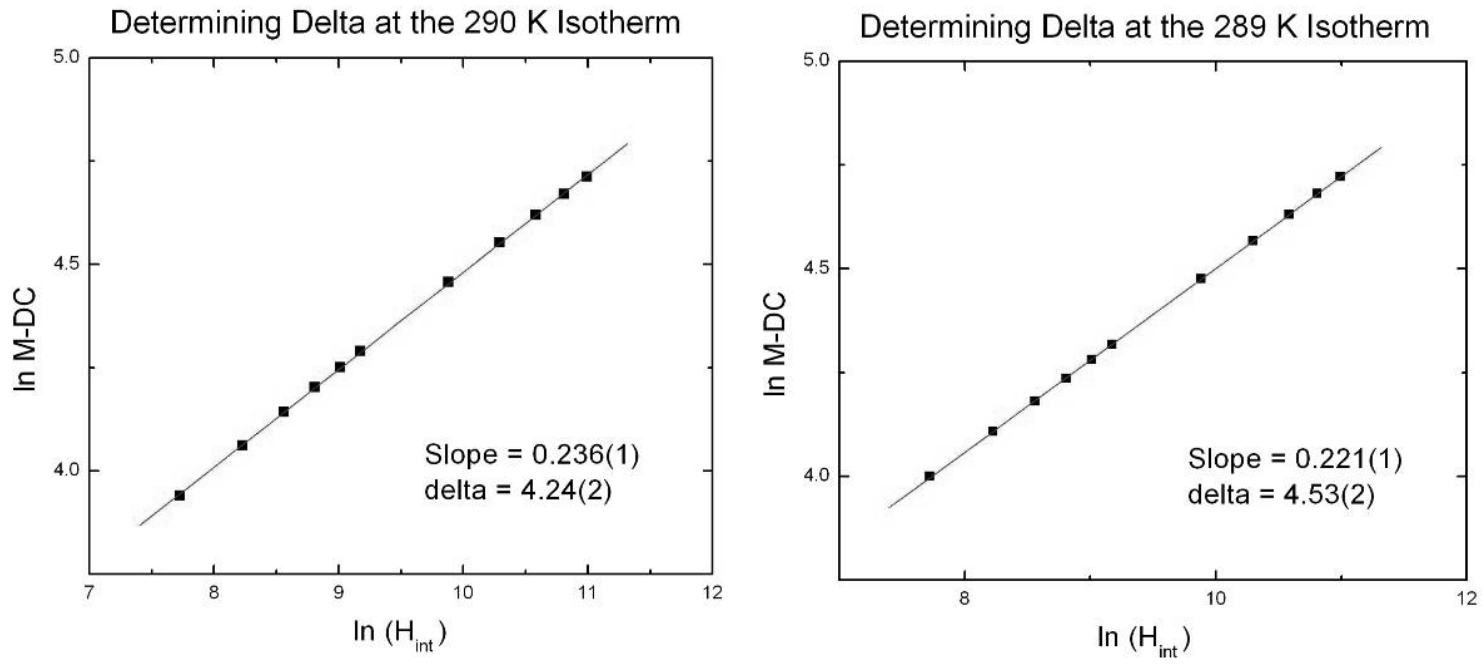

Figure 5. Determining Delta on the critical isotherm. 


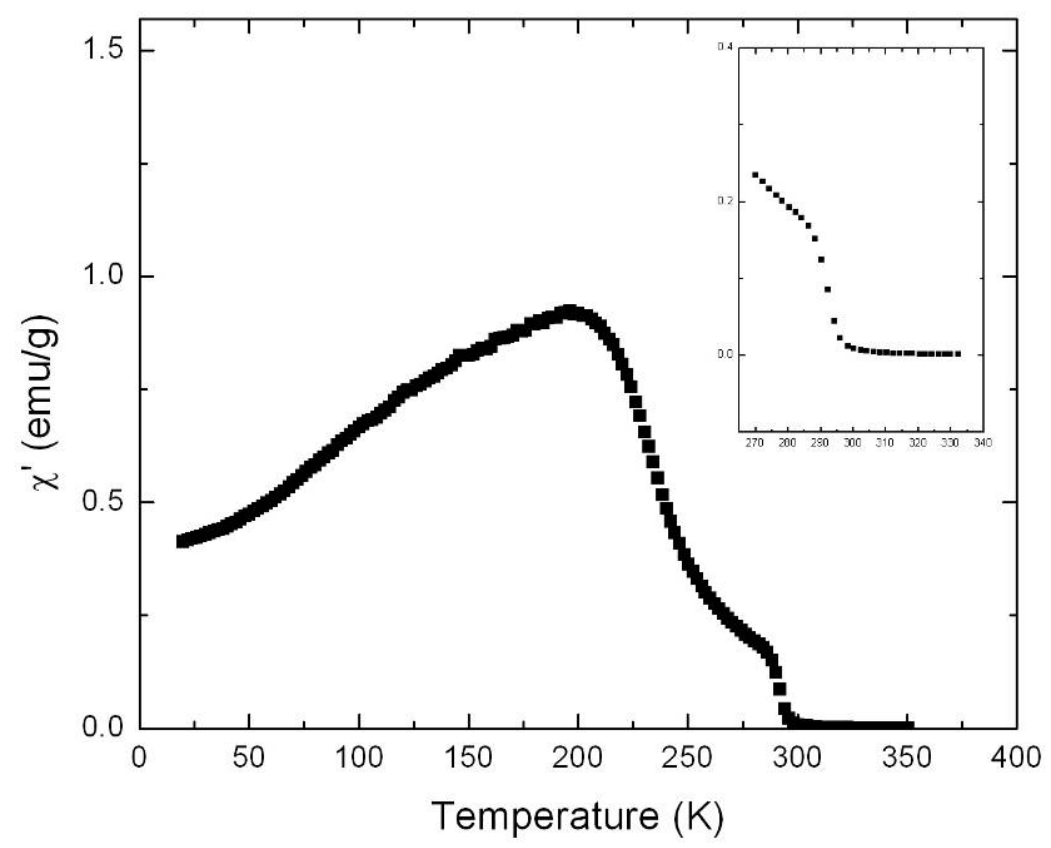

Figure 6. AC susceptibility as a function of temperature $(20 \mathrm{~K} \leq \mathrm{T} \leq 350 \mathrm{~K})$.

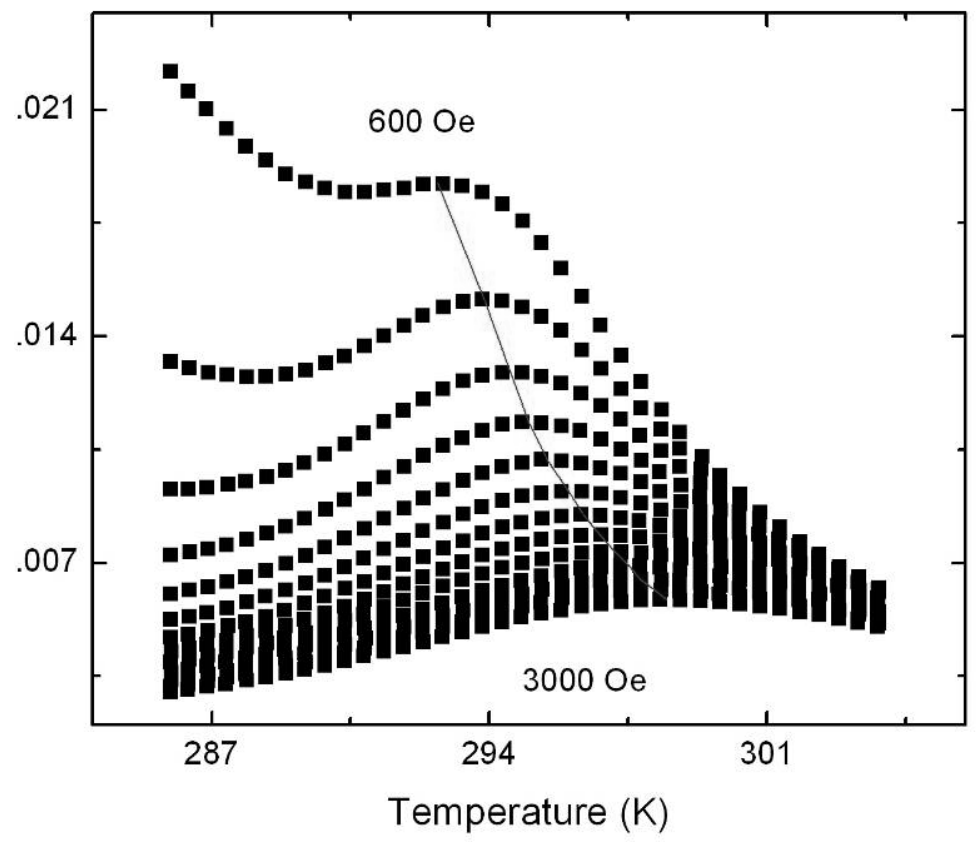

Figure 7. AC susceptibility versus Temperature for DC bias fields for temperatures near the transition temperature. Bias fields range from 600 Oe to 3000 Oe in intervals of $200 \mathrm{Oe}$. The gray line on the graph passes through the maxima.

Plots of $\ln \left(X^{\prime}\right)$ vs. the $\ln \left(\varepsilon_{m}\right)$ can thus provide another measurement of $\gamma$. The data in Figure 9 is linear for applied fields greater than $1000 \mathrm{Oe}$, and its slope results in $\gamma=$ $1.308 \pm 0.012$, corroborating the exponent value obtained earlier. 


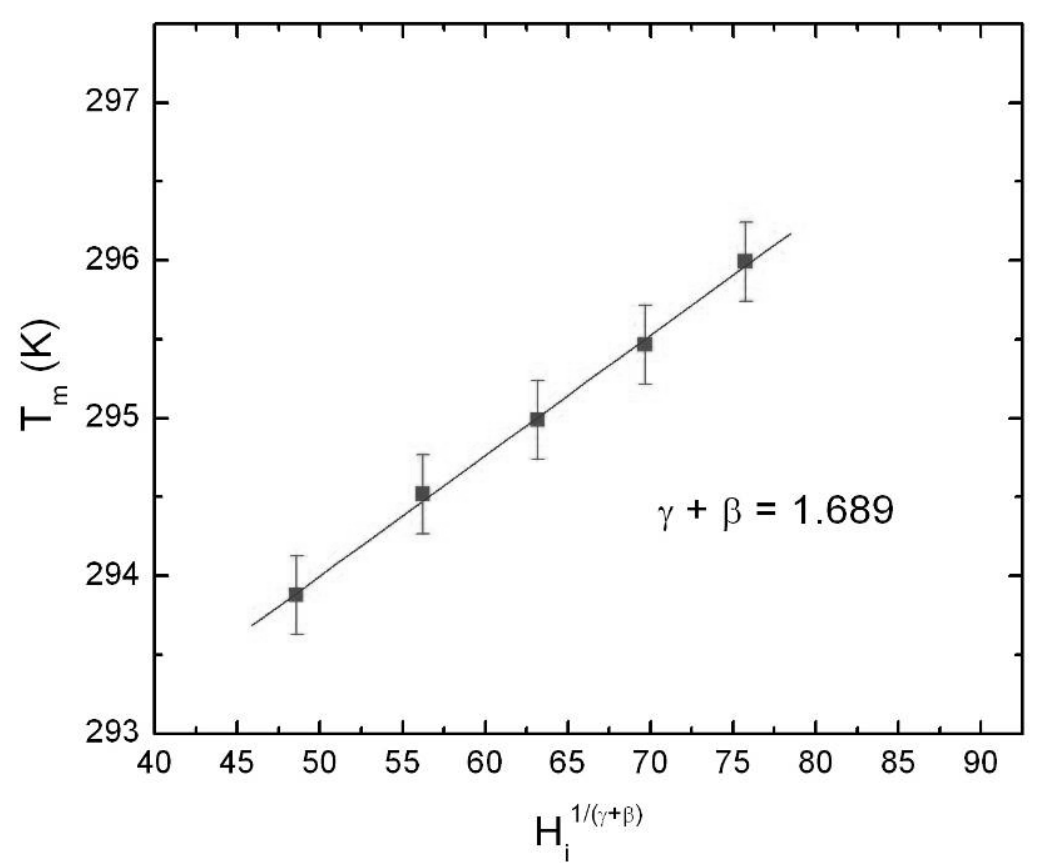

Figure 8. $T_{m}$ versus $H^{(1 / \gamma+\beta)}$. The intercept on the vertical axis gives a measure of $T_{C}$.

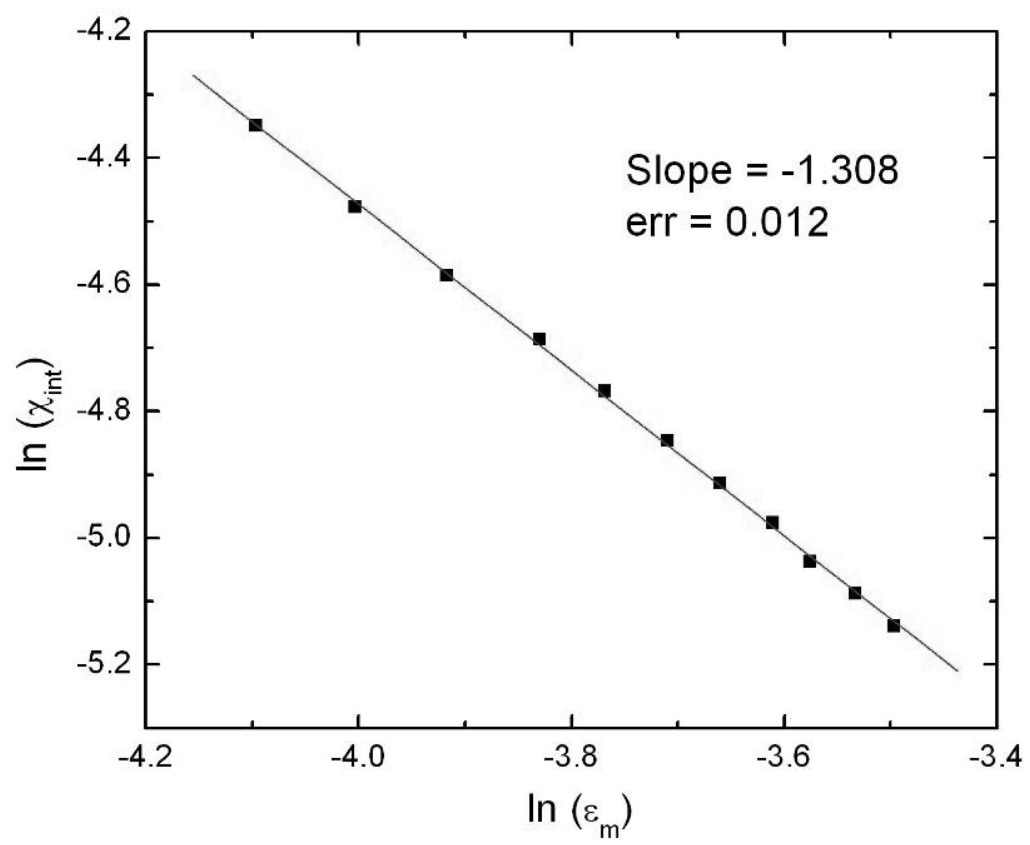

Figure 9. Log-Log plot of internal susceptibility versus reduced temperature, $\varepsilon_{m}$. $\gamma$ is equal to the negative of the slope.

\section{DISCUSSION}

The critical-exponent values for nanostructured gadolinium, bulk crystalline gadolinium, and other amorphous gadolinium based ferromagnets [12], as well as some theoretical values for the exponents, are included in Table 1 for comparison.

The exponents of melt-spun $\mathrm{Gd}$ have shifted away from the bulk gadolinium 


\begin{tabular}{cccccc}
\hline Material & Ref. & $\boldsymbol{\beta}$ & $\mathbf{Y}$ & $\boldsymbol{\delta}$ & $\mathbf{T}_{\mathbf{C}}(\mathrm{K})$ \\
Nanostructured Gd Sample & $\begin{array}{c}\text { This } \\
\text { work }\end{array}$ & $0.389 \pm 0.017$, & $1.300 \pm 0.014$, & $4.32 \pm 0.02$ & $289.70 \pm 016$ \\
& & & & & \\
Crystalline Gadolinium (Bulk) & {$[11]$} & $0.381-0.40$ & $1.196-1.24$ & $3.615(2)$ & $294(1)$ \\
Amorphous Gd-TM Ferromagnets & {$[9]$} & $0.34-0.44$ & $1.16-1.29$ & $3.6-3.96$ & - \\
& & & & & \\
3D Heisenberg Model (Theory) & {$[9]$} & $0.365(25)$ & $1.386(4)$ & $4.80(4)$ & - \\
Mean Field Theory & {$[10]$} & 0.5 & 1 & 3 & - \\
\hline
\end{tabular}

Table 1. Comparisons of parameters of Pure Melt-Spun Gadolinium Sample with experimental and theoretical values reported in the literature.

exponents and toward the short-range 3D Heisenberg model exponents, indicating that the long-range interactions in $\mathrm{Gd}$ are being suppressed. The decrease in $\mathrm{T}_{\mathrm{C}}$ relative to bulk $\mathrm{Gd}$ supports this as well, signifying that nanocrystalline gadolinium is distinct from its bulk form. The melt-spun gadolinium may exhibit more short-range-like interactions because the amorphous phase at the boundaries of the nanocrystals is frustrating the long-range interactions that normally direct the transition. Note that the value of the exponent $\beta$ remains rather close to that of bulk crystalline $\mathrm{Gd}$ and also to the theoretical value for the 3D Heisenberg model indicating unambiguously that in the range of temperatures used for our analysis, the $\mathrm{Gd}$ magnetic moments behave as three dimensional vectors.

\section{CONCLUSIONS}

We have studied the critical behavior of nanostructured gadolinium using a combination of Arrott-Noakes analysis and AC susceptibility scaling analysis, which has indicated a paramagnetic to ferromagnetic transition does take place at a Curie temperature $\mathrm{T}_{\mathrm{C}}=289.70 \pm 0.16 \mathrm{~K}$. The resulting critical exponents are $\gamma=1.300 \pm$ $0.014, \beta=0.389 \pm 0.017$, and $\delta=4.32 \pm$ 0.02. Comparison with the critical exponents of bulk $\mathrm{Gd}$ shows that meltspinning has created a two-phased nanocrystalline system that has to some extent suppressed the long range interactions normally present in $\mathrm{Gd}$. The structural inhomogeneity has also caused the $T_{C}$ to decrease as well. The results reported here are also part of an ongoing study of disordered magnetic systems and will be used as a reference for melt-spun gadolinium-iron alloys that are yet to be tested.

\section{ACKNOWLEDGEMENTS}

This work was funded by National Science Foundation awards DMR-504177 and DMR0504706.

\section{REFERENCES}

1. E. Kita, Y. Hata, K. Yano, H. Suzuki, G. Kido. J. Appl. Phys., 95 (2004) 68346836.

2. T.M. Danh, N.H. Duc, N.P. Thuy. J. Magn. Magn. Mater. 185 (1998) 105108.

3. K. Yano, Y. Akiyama, K. Tokumitsu, E. Kita, H. Ino. J. of Magn. Magn. Mater. 214 (2000) 217-224.

4. V. Petkov, K. Yano, E. Kita. Phys. Stat. sol. 157 (1996) 365-372.

5. K. Yano, K. Kita, K. Tokumitsu, H. Ino, A. Tasaki. J. Magn. Magn. Mater. 104107 (1992) 131-132.

6. K. Yano, K. Tokumitsu, E. Kita, H. Ino, A. Tasaki. Japanese J. Appl. Phys., 30 (1991) L 482 - L 485.

7. M. Sahana, U.K. Rößler, N. Ghosh, S. Elizabeth, H.L. Bhat, K. Dorr, D. Eckert, M. Wolf, K.H. Müller. Phys. Rev. B 68 (2003) 144408.

8. A. Arrott, J. Noakes, Phys. Rev. Lett. 19 (1967) 786-789.

9. S. N. Kaul, J. Magn. Magn. Mat. 53 (1985) 5. 
10. M. Plischke, B. Bergersen. Equilibrium Statistical Physics, $2^{\text {nd }}$ Ed. (1994) Singapore: World Scientific Publishing Co.

11. J. H. Zhao, H. P. Kunkel, X. Z. Zhou, G.
Williams, and M. A. Subramaniam. Phys. Rev. Lett. 83 (1999) 219.

12. S. Dan'kov, A. M. Tishin, V. K. Pecharsky, K. A. Gschneidner Jr. Phys. Rev. B 57 (1998) 3478-3490

\section{Center for Education in Nanoscience \&. Nanotechnology \\ University of Northern lowa - Cedar Falls • lowa • USA \\ A federally-funded initiative to educate a high-tech professional workforce in nanoscience and nanotechnology}
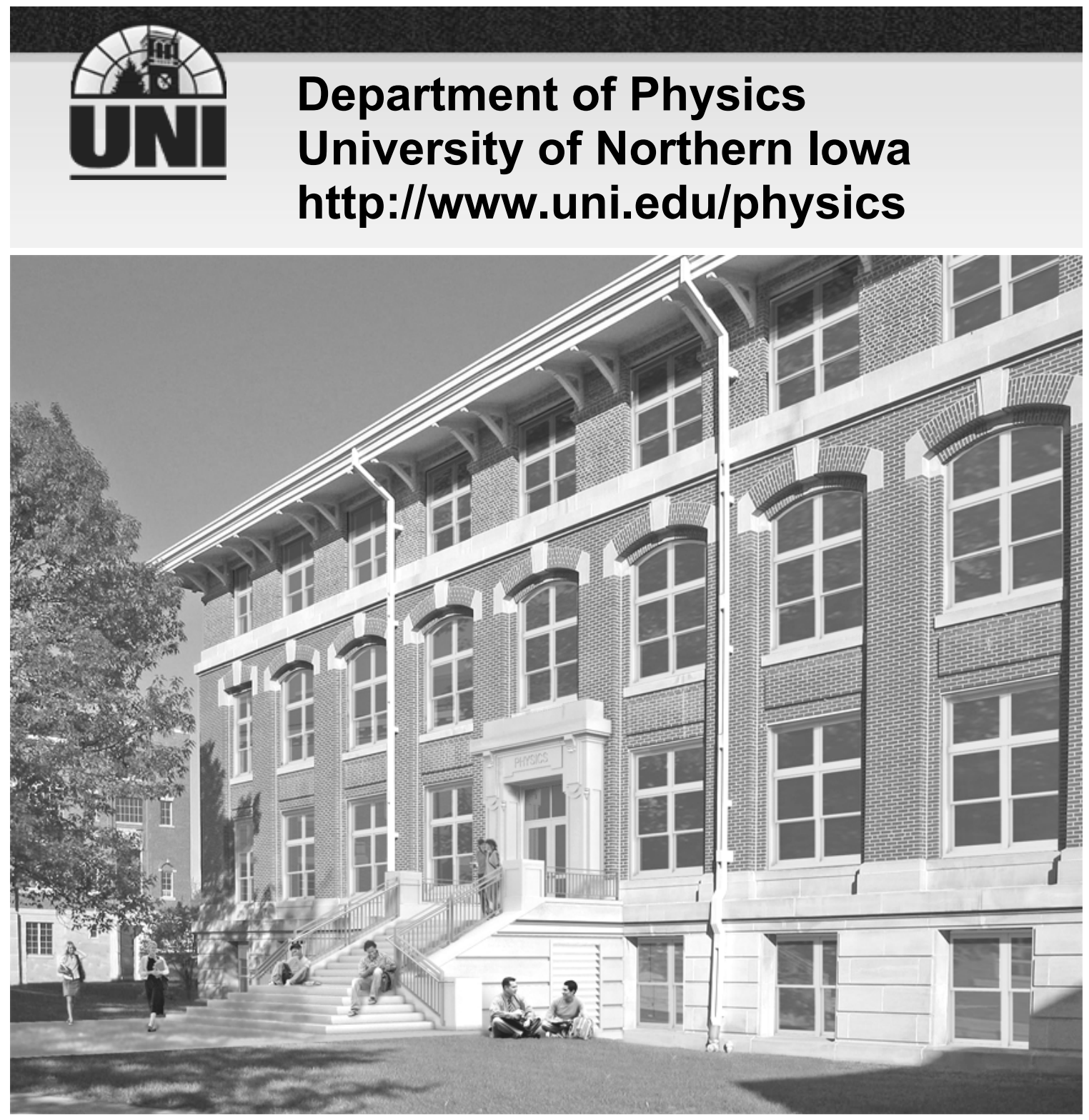


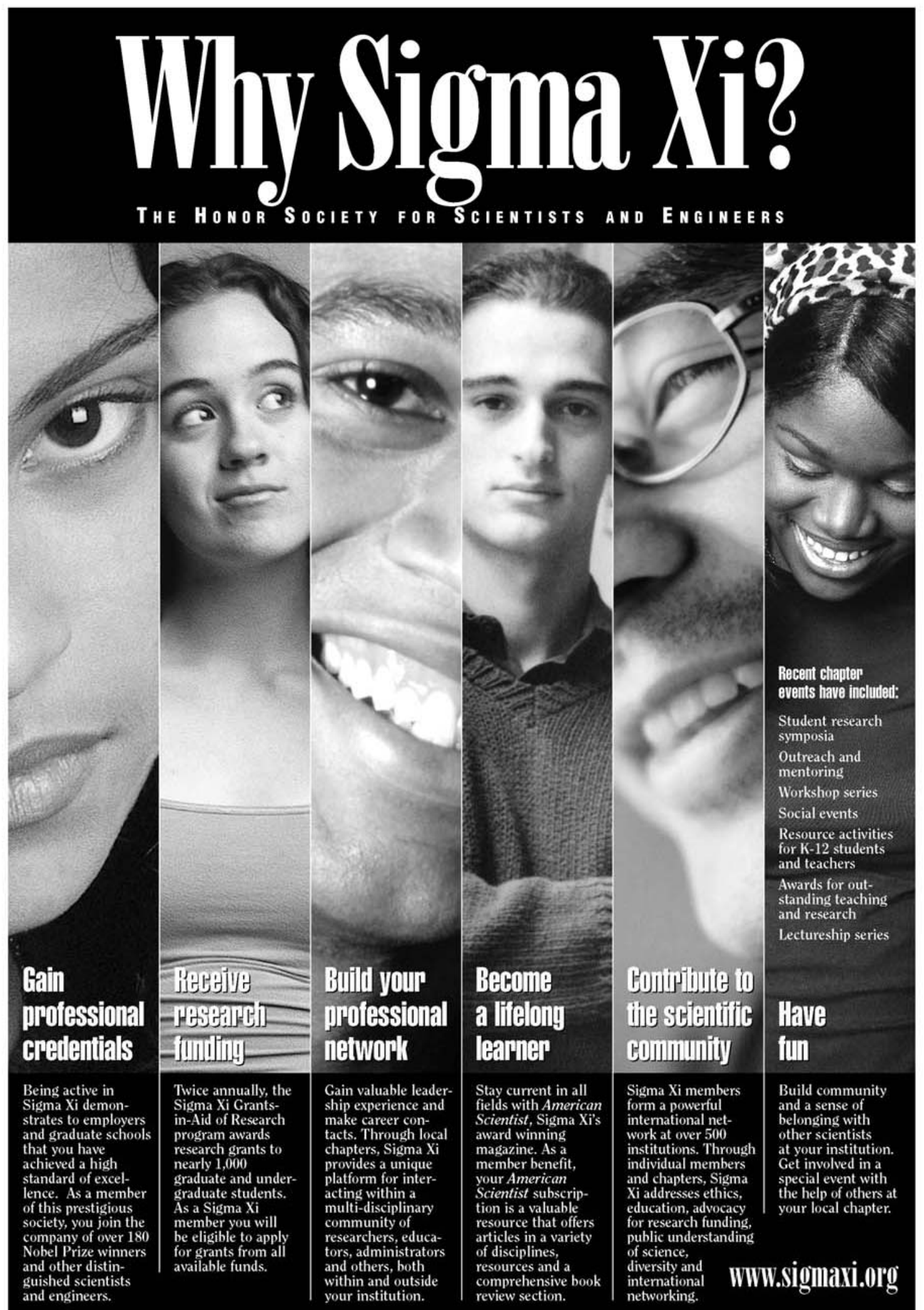

Sigma Xi, The Scientific Research Society • 98 Alexanter Drive • P.O. Box 13975 • Research Triangle Park, NC 27708 • 818-549-4691 • 800-243-6534 


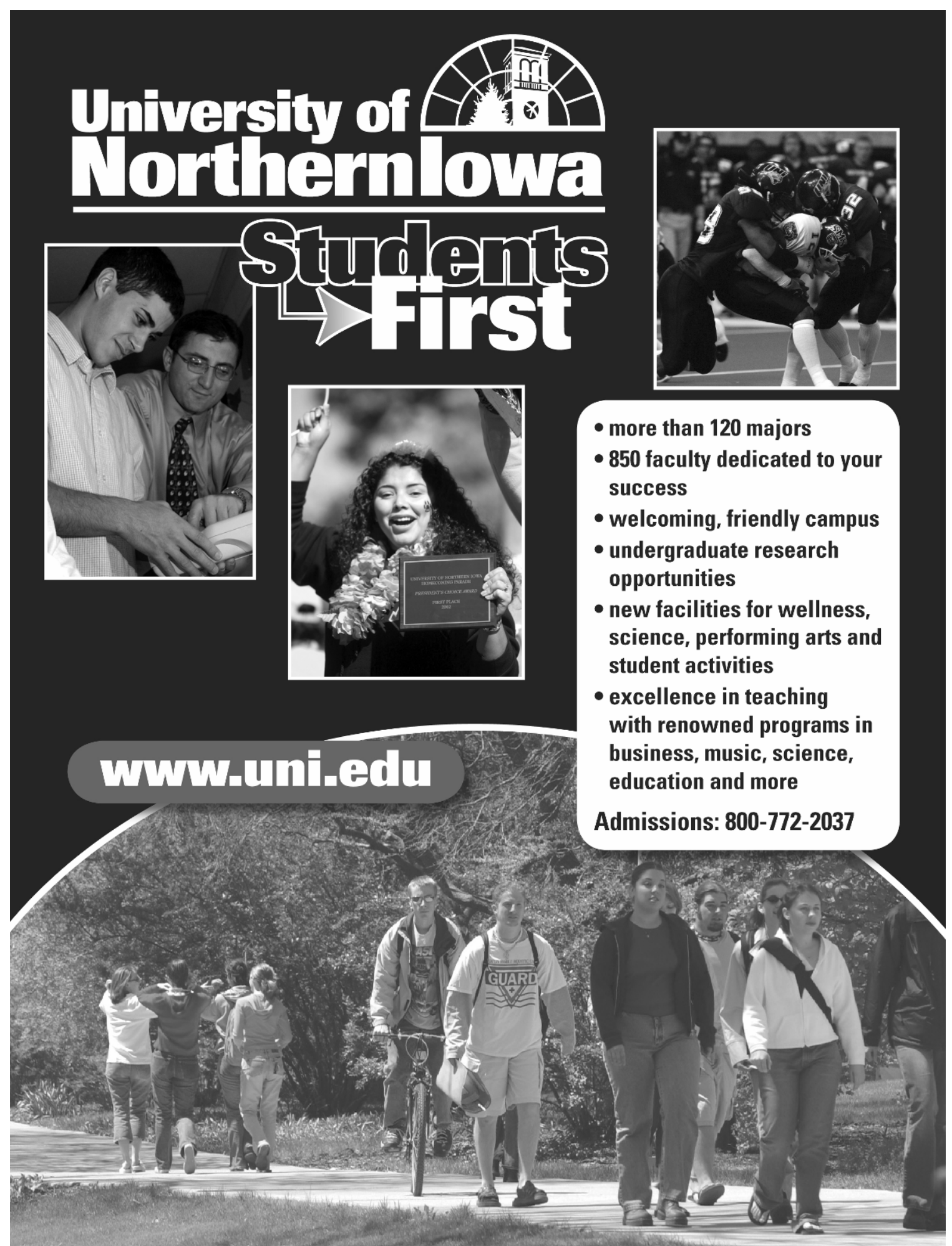




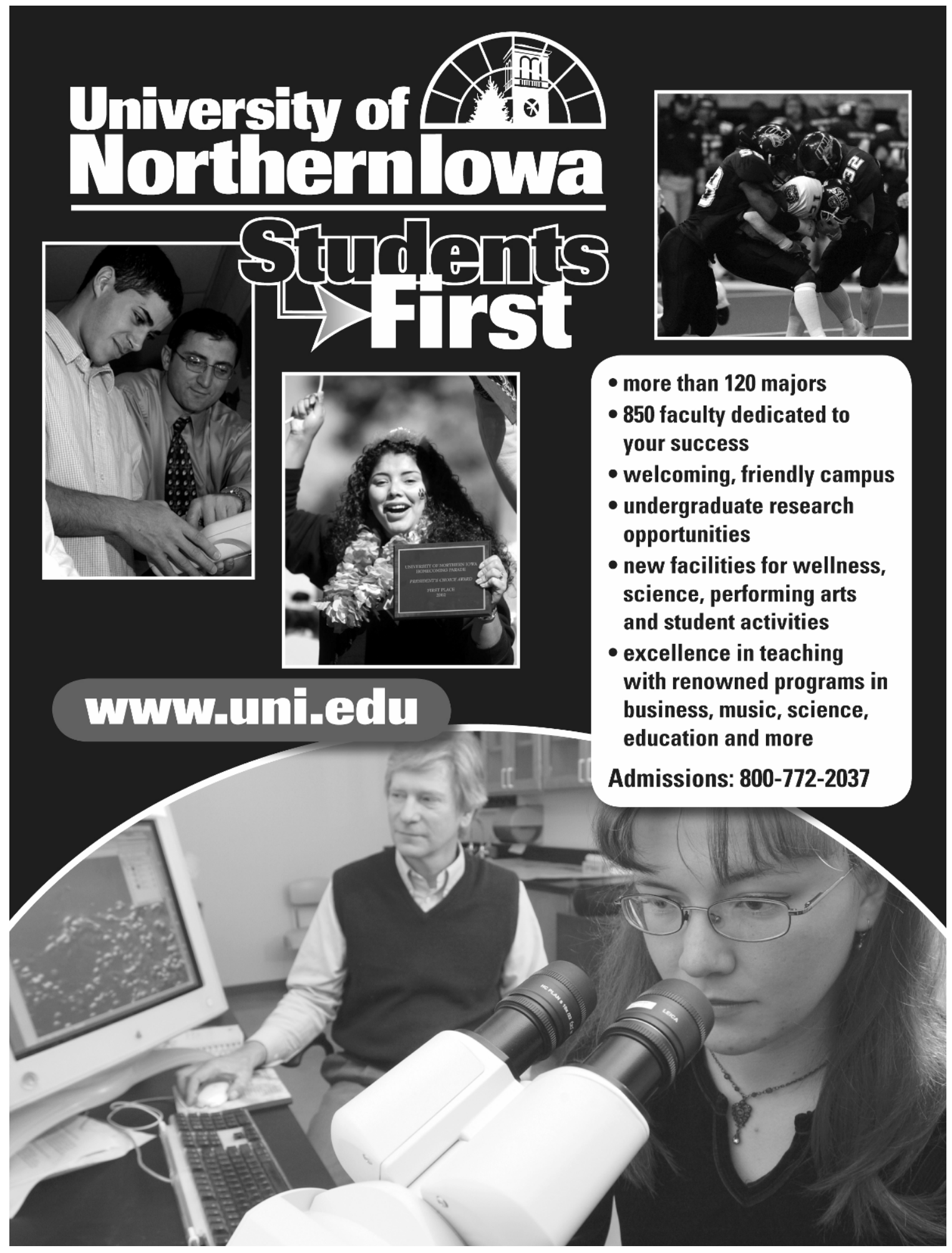

